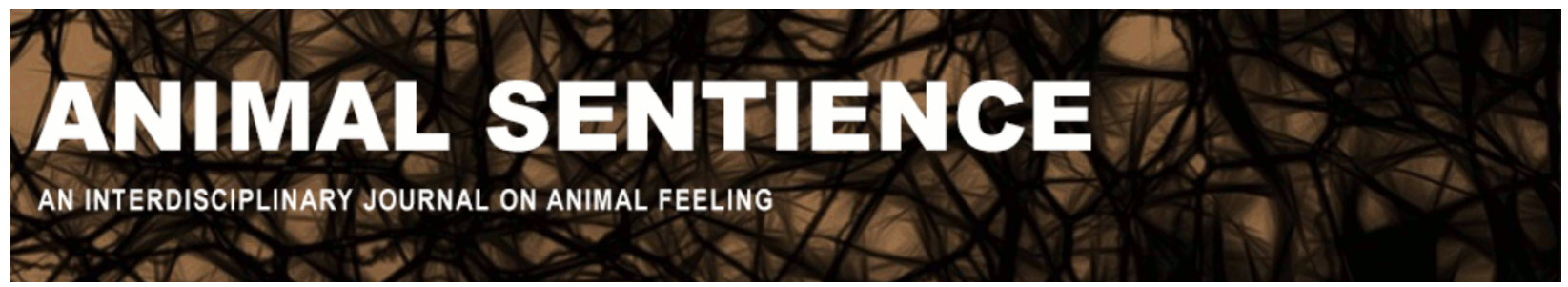

Elwood, Robert W. (2016) A single strand of argument with unfounded conclusion. Animal Sentience 3(19)

DOI: $10.51291 / 2377-7478.1056$

Date of submission: 2015-11-02

Date of acceptance: 2015-12-13 (c) 


\title{
A single strand of argument with unfounded conclusion
}

Commentary on Key on Fish Pain

\author{
Robert W. Elwood \\ School of Biological Sciences \\ Queen's University, Belfast
}

\begin{abstract}
Key (2016) describes the neural system involved in human pain experience in an excellent fashion but then suggests that only that complete system can generate the experience of pain. Thus animals without all components will not feel pain. This argument has been refuted in the past by analogy to vision where it is clear that a broad range of taxa, vertebrate and invertebrate, have good visual abilities albeit with completely different central nervous systems and receptors. This known counterargument to Key's main idea is not mentioned in the target article. Further criteria that might indicate pain and studies examining these criteria in fish and other animals also get scant attention. The function of pain in fitness terms is not considered, yet that might provide clues as to when and how often pain might have evolved.
\end{abstract}

Robert W. Elwood R.Elwood@qub.ac.uk is Emeritus Professor of Animal Behaviour at Queen's University, Belfast. His research is on immediate and prolonged changes in behaviour and physiology after noxious stimulation in crustaceans and whether they are consistent with the idea of pain.

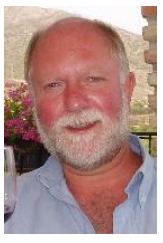
http://www.qub.ac.uk/schools/SchoolofBiologicalSciences/People/ ProfessorRWElwood/

The target article by Key (2016) provides an excellent review of the neuronal structures implicated in pain experiences in humans. He notes that with the destruction of particular aspects of the system, different aspects of pain experience are modified or lost completely. This review is the main strength of the paper. However, Key then relies on a single strand of evidence and argument to conclude that fish have no feeling of noxious stimuli. Key contends that only the specific and complete human system for pain can generate the experience of pain. Thus, any taxon that lacks the neuronal system or even just one part of that system will be incapable of feeling pain. This is a sweeping anthropocentric argument that ignores other modalities such as vision. The neuronal structures involved in human visual perception are also well known. It is clear, however, that other taxa lack specific aspects of the system or, in the case of invertebrates, have very different structures based on an entirely different CNS. Thus, according to the argument proposed by Key, these taxa, lacking the human visual system, must be blind. The fact that even though arthropods, molluscs and fish have neural systems that are very different from one another as well as from humans, they all nevertheless have visual abilities indicates that very different neuronal systems can serve a common function. This often repeated argument (e.g. Elwood 2011) is ignored in Key's review. This is a pity because the argument severely undermines the single strand of argument in the target article, and it should have been addressed. 
Further, pain is said to have survival value only if it is "mapped to specific body locations," and there is an excellent description of human neuronal structures that appear to implement this ability. Nothing is said about fish or other animals having an ability to map to specific body locations. For example, fish injected with acetic acid into the lip will rub the lip on the substrate (Sneddon et al. 2003), and hermit crabs (Elwood \& Appel 2009) and prawns (Barr et al. 2008) will rub the specific afflicted areas indicating some awareness of which part of the body has been subject to noxious stimulation. Thus, again we see animals that lack the specific neuronal structure nevertheless exhibiting the same ability as humans. It would have been useful to have some discussion of the localization of the stimulus site in other animals. Instead, Key tries to challenge the idea of pain in fish by citing studies that report learning in the spinal cord of mammals as well as the ability of dogs with a spinal block to localise the site of stimuli on the body. Thus, having described both localisation of the stimulus and learning as key aspects of pain, Key suggests that they do not require a brain.

There is still much else that is missing from this review, however. There is virtually no mention of evidence that is consistent with the interpretation of pain in fish or other taxa. There is no mention of papers that examine criteria other than similarity of neuronal structures that might nevertheless be consistent with pain experience in animals (Bateson 1991; Sneddon et al. 2014). There is also no consideration of the function of pain in terms of improved fitness from having this experience rather than pure nociception and reflexive response. Clearly, nociception is a vital sensory and responsive system that provides protection from damaging stimuli. It enables animals to move all of the body or just the inflicted part away from the source of potential tissue damage. What added value does feeling pain offer in terms of improved fitness? The human system is large and complex and will have costs of production during ontogeny and during use. For selection to have developed the ability to feel pain, those costs must be offset by substantial improvements in survival and reproduction. That is, the unpleasant experience that we call pain must be good for us and, presumably, for other animals too. By considering the adaptive function of pain, we might gain insights into when that ability arose during evolution. For example, we might ask whether there is any reason why an aquatic habitat or some other aspect of an aquatic species' environment or life-history might preclude any fitness advantage of pain.

I was also concerned with some of Key's terminology. For example, the statement that patients could all recognize "a painful stimulus as painful" is clearly tautological. I am not fond of the use of terms like "perceive pain" because that suggests (at least to me) that pain is something to do with perception of external stimuli rather than a feeling generated within the brain. I was puzzled by the idea that the particular neuronal structures are required for "pain related computations," but I am not sure what computations might be required.

Finally, although Key acknowledges in the introduction that absence of evidence does not mean an absence of pain (in fish) and later on that it is not possible to prove that fish do not feel pain, the final conclusion is, nevertheless, dogmatic. "What then do noxious stimuli feel like to a fish? The evidence best supports the idea that they don't feel like anything to a fish." That is, fish are not aware of tissue damage because they lack one or more specific neuronal structures found in humans. It is difficult to support such a conclusion on the evidence and argument adduced in the target article. 


\section{References}

Barr, S., Laming, P.R., Dick, J.T.A. \& Elwood, R.W. (2008) Nociception or pain in a decapod crustacean? Animal Behaviour, 75, 745-751.

Elwood, R.W. (2011) Pain and suffering in invertebrates? ILAR Journal, 52, 175-184.

Elwood, R. W. \& Appel, M. (2009) Pain in hermit crabs? Animal Behaviour 77, 1243-1246.

Key, B. (2016). Why fish do not feel pain. Animal Sentience 2016.003.

Sneddon L.U., Braithwaite V.A. \& Gentle, M.J. (2003) Do fishes have nociceptors? Evidence for the evolution of a vertebrate sensory system. Proceeding of the Royal Society of London B. 270, 1115-1121. 